\title{
A Regular Boundary Element Method for Fluid Flow
}

Link to publication record in Manchester Research Explorer

\section{Citation for published version (APA):}

Patterson, C., \& Sheikh, M. (1982). A Regular Boundary Element Method for Fluid Flow. International Journal for Numerical Methods in Fluids, 2(0), 239-251.

\section{Published in:}

International Journal for Numerical Methods in Fluids

\section{Citing this paper}

Please note that where the full-text provided on Manchester Research Explorer is the Author Accepted Manuscript or Proof version this may differ from the final Published version. If citing, it is advised that you check and use the publisher's definitive version.

\section{General rights}

Copyright and moral rights for the publications made accessible in the Research Explorer are retained by the authors and/or other copyright owners and it is a condition of accessing publications that users recognise and abide by the legal requirements associated with these rights.

\section{Takedown policy}

If you believe that this document breaches copyright please refer to the University of Manchester's Takedown Procedures [http://man.ac.uk/04Y6Bo] or contact uml.scholarlycommunications@manchester.ac.uk providing relevant details, so we can investigate your claim.

\section{OPEN ACCESS}




\title{
A REGULAR BOUNDARY ELEMENT METHOD FOR FLUID FLOW
}

\author{
C. PATTERSON† AND M. A. SHEIKH \\ Department of Mechanical Engineering, University of Sheffield, \\ Mappin Street, Sheffield S1 3JD, U.K.
}

\begin{abstract}
SUMMARY
The Boundary Element Method is now well established as a valid numerical technique for the solution of field problems, equal to the Finite Element Method in generality and surpassing it in computational efficiency in some cases. ${ }^{1}$ In this paper is presented a 'Regular Boundary Element Method' as applied to inviscid laminar fluid flow problems. It involves the formation of a system of regular integral equations obtained by moving the singularity outside the domain of the given problem. It is also shown that non-conforming elements may be used whereby freedoms are not defined at the geometric nodes under the boundary element discretization. A linear element is developed here; higher order variants could easily be defined. Satisfactory numerical results have been obtained using the proposed regular method with both conventional (continuous across the boundary) and non-conforming boundary elements for two-dimensional inviscid laminar fluid flow problems having regular and singular solutions.
\end{abstract}

KEY wORDs Regular Discontinuous Boundary Elements Fluid Flow

\section{INTRODUCTION}

The Boundary Element Method has evolved slowly over more than a decade but until recently it has not received widespread attention. A basic reason for this is that the resulting linear algebraic equations are non-symmetric and, on a simple approach, fully populated. In consequence, it has not appeared as an attractive competitor to the Finite Element Method.

However, the abundant success of the Finite Element Method has led to progressively more exacting demand upon it. Particularly, whereas previously designers were content with a simplified two-dimensional approach, there is now an increasing demand for more detailed three-dimensional analyses. This is especially so in high risk contexts, such as nuclear power.

The increased computing overhead in going from two to three dimensions is considerable so that there is some urgency in exploring the methods which may be more efficient than the Finite Element Method in three dimensions. Because of the square-cube relation of degrees of freedom, any boundary domain method is attractive in this light.

In its usual form the Boundary Element Method starts from the infinite system of boundary integral equations obtained from the governing field equations by appropriate integration by parts and employing a fundamental solution as kernel. ${ }^{2}$ This system is truncated to a finite system on selection of a finite number of fundamental solutions and discretized after the manner of the Finite Element Method. Non-symmetric, full, linear algebraic equations ensue.

$\uparrow$ Senior Lecturer.

+ Postgraduate Student.

0271-2091/82/030239-13\$01.30

Received 24 August 1981

(C) 1982 by John Wiley \& Sons, Ltd.

Revised 23 November 1981 
There are two drawbacks in the method as normally used. Firstly, the singular point of the fundamental function is taken on the boundary of the problem, thereby giving a system of singular integrals. This raises two problems:

(1) Not only does the accurate evaluation of these singular integrals require careful and special treatment in the neighbourhood of the singular point but it may also contribute to relatively higher computational cost.

(2) The class of problems for which the method is well defined, may be unduly restrictive because of divergence of the integrals.

It has been shown that there is no essential reason for following this approach, ${ }^{3}$ and that the singular point may validly be taken outside the domain of the problem resulting in a system of regular integral equations. ${ }^{4}$

Secondly, as with Finite Elements, a hierarchy of boundary elements may be defined, starting with a constant element and ranging through linear, quadratic elements etc. Presumably, in analogy with Finite Elements, inter-element continuity of the unknown functions has been imposed in applications. This continuity gives rise to at least three problems: ${ }^{5}$

(1) At a point where the surface is not smooth, the normal is not defined but the freedom there demands a valid normal.

(2) At an interface where there is a change in the nature of boundary conditions (say between potential and its derivative) apparently both types of freedoms are constrained.

(3) When the problem is partitioned into subregions, ${ }^{6}$ there can be excessive constraint where several surfaces meet.

Accommodation to the second and third problems has been devised by appropriate suppression of degrees of freedoms and to the first by the introduction of two freedom nodes close to the geometric singularity on either side. The last approach is objectionable since, if the nodes are not closely spaced the boundary integrals are not well discretized whereas if they are, the resulting algebraic equations are ill-conditioned. This is because proximity of freedom nodes implies linear dependence in the algebraic equations. The root of the problem is interelement continuity. This is necessary with Finite Elements ${ }^{7}$ to ascertain positive definiteness of the operator of the governing equations but it is not a requirement in the Boundary Element Method. The latter point is amply borne out by the success of constant elements. It has been demonstrated that non-conforming elements ${ }^{8}$ obviate these problems. $^{9}$

In this paper the 'Regular Boundary Integral Equations' are presented and both conventional and non-conforming linear boundary elements are used in discretizing the system. Two two-dimensional inviscid, laminar, fluid flow problems are examined. The convergence behaviour of the approximate solution for a singular problem with regard to mesh densities and its sensitivity to location of fundamental solution singularity is appraised.

\section{THEORY}

Consider a Potential Function $\phi$ over the domain $\Omega$, of the given problem which satisfies the governing (Laplace's) equation:

$$
\nabla^{2} \phi=0 \text { in } \Omega
$$




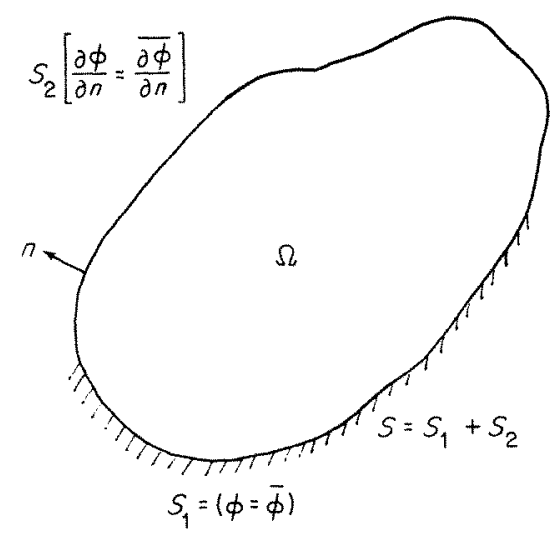

Figure 1. Problem definition

The boundary conditions for the problem are (Figure 1)

(a)

$$
\begin{aligned}
\phi & =\bar{\phi} \text { on } S_{1} \text { (Essential condition) } \\
\frac{\partial \phi}{\partial n} & =\frac{\partial \phi}{\partial n} \text { on } S_{2} \text { (Natural condition) }
\end{aligned}
$$

where the total boundary is given as $S=S_{1}+S_{2}$.

Introducing a weighting function $\phi^{*}$ which has continuous first derivatives and which satisfies the governing equation, we can write the weighted residual statement as:

$$
\int_{\Omega}\left(\nabla^{2} \phi\right) \phi^{*} \mathrm{~d} \Omega=\int_{S_{2}}\left(\frac{\partial \phi}{\partial n}-\frac{\overline{\partial \phi}}{\partial n}\right) \phi^{*} \mathrm{~d} S-\int_{S_{1}}(\phi-\bar{\phi}) \frac{\partial \phi^{*}}{\partial n} \mathrm{~d} S
$$

Integrating the Laplacian term of equation (3) by parts twice, we obtain:

$$
\int_{\Omega}\left(\nabla^{2} \phi^{*}\right) \phi \mathrm{d} \Omega=-\int_{S_{2}} \frac{\overline{\partial \phi}}{\partial n} \phi^{*} \mathrm{~d} S-\int_{S_{1}} \frac{\partial \phi}{\partial n} \phi^{*} \mathrm{~d} S+\int_{S_{2}} \phi \frac{\partial \phi^{*}}{\partial n} \mathrm{~d} S+\int_{S_{1}} \bar{\phi} \frac{\partial \phi^{*}}{\partial n} \mathrm{~d} S
$$

Assuming a concentrated source at a point $i$, the governing equation is

$$
\nabla^{2} \phi^{*}+\Delta^{i}=0
$$

where $\Delta^{i}$ is the Dirac delta function for which,

$$
\int_{\Omega} \phi\left(\nabla^{2} \phi^{*}+\Delta^{i}\right) \mathrm{d} \Omega=\int_{\Omega} \phi \nabla^{2} \phi^{*} \mathrm{~d} \Omega+\phi^{i}
$$

If equation (5) is to be satisfied by the fundamental solution then:

$$
\int_{\Omega} \phi\left(\nabla^{2} \phi^{*}\right) \mathrm{d} \Omega=-\phi^{i}
$$

and hence equation (4) becomes:

$$
\phi^{i}+\int_{S_{1}} \bar{\phi} \frac{\partial \phi^{*}}{\partial n} \mathrm{~d} S+\int_{S_{2}} \phi \frac{\partial \phi^{*}}{\partial n} \mathrm{~d} S=\int_{S_{1}} \frac{\partial \phi}{\partial n} \phi^{*} \mathrm{~d} S+\int_{S_{2}} \frac{\overline{\partial \phi}}{\partial n} \phi^{*} \mathrm{~d} S
$$

If $\bar{\phi}$ and $\partial \bar{\phi} / \partial n$ values are known for respective parts of the boundary, we can write 
equation (8), in general, for the total boundary as:

$$
c^{i} \phi^{i}+\int_{S} \phi \frac{\partial \phi^{*}}{\partial n} \mathrm{~d} S=\int_{S} \frac{\partial \phi}{\partial n} \phi^{*} \mathrm{~d} S
$$

in which $\phi^{*}$ is the fundamental solution and $c^{i}$ is the unknown coefficient. $\phi^{*}$ for the isotropic case in two dimensions is given as $\phi^{*}=\frac{1}{2 \pi} \ln (1 / r)$ where $r$ is the distance between the source and the point under consideration.

If the said point $i$ is located outside the domain of the given problem, this coefficient $c^{i}$ equals zero and equation (9) becomes:

$$
\int_{S} \phi \frac{\partial \phi^{*}}{\partial n} \mathrm{~d} S=\int_{S} \frac{\partial \phi}{\partial n} \phi^{*} \mathrm{~d} S
$$

Equation (10) forms the basis of 'Regular Boundary Integrals' thereby giving a system of equations; one for each singular point corresponding to the 'Freedom node' under the boundary element discretization and located outside the domain of the problem. These Regular Equations can also be obtained when the domain of the given problem is partitioned into sub-regions in which case the singular points corresponding to the nodes on the interfaces do not lie in the solution zone as the sub-regions are disjoint at the time of setting up the equations.

\section{CONSTANT BOUNDARY ELEMENTS}

The boundary is discretized into $N$ elements (say) and the values of potential and its derivative are assumed to be constant on each element, and equal to the value at the mid-node of the element. The singular point corresponding to this mid-node $i$ is located at an arbitrary distance from $i$ and along the positive normal (Figure 2).

Equation (10) for this singular point corresponding to the boundary node $i$ becomes in discretized from:

$$
\sum_{j=1}^{N} \phi_{j} \int_{S_{j}} \frac{\partial \phi^{*}}{\partial n} \mathrm{~d} S=\sum_{i=1}^{N}\left(\frac{\partial \phi}{\partial n}\right)_{j} \int_{S_{j}} \phi^{*} \mathrm{~d} S
$$

The integrals in equation (11) can be evaluated numerically for all segments over the boundary including the one containing node $i$. $N$ such equations are obtained and solved for

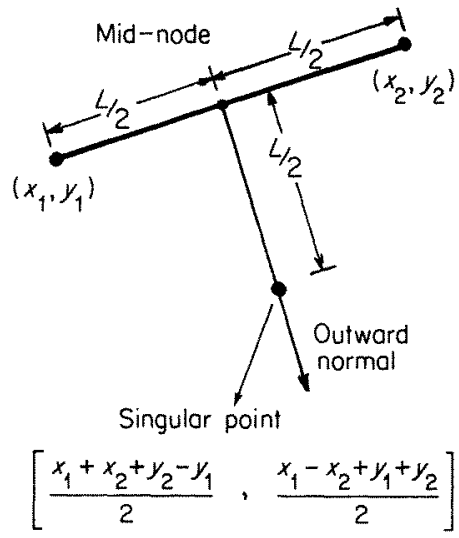

Figure 2. Location of singular point for constant boundary element 
the boundary unknowns. Once the solution over the whole boundary is obtained the interior solution can be generated using equation (9) with $c^{i}$ equal to unity.

\section{LINEAR BOUNDARY ELEMENTS}

The variation of $\phi$ and $\partial \phi / \partial n$ is assumed to be linear within each element. For $N$ elements, equation (10) can be written as:

$$
\sum_{j=1}^{N} \int_{S_{\mathrm{j}}} \phi \frac{\partial \phi^{*}}{\partial n} \mathrm{~d} S=\sum_{j=1}^{N} \int_{S_{j}} \frac{\partial \phi}{\partial N} \phi^{*} \mathrm{~d} S
$$

The values of $\phi$ and $\partial \phi / \partial n$ at any point of an element can be written in terms of their nodal values and interpolation functions $F_{1}$ and $F_{2}$ as:

$$
\begin{aligned}
\phi(\xi) & =F_{1} \phi_{1}+F_{2} \phi_{2} \\
\frac{\partial \phi}{\partial n}(\xi) & =F_{1}\left(\frac{\partial \phi}{\partial n}\right)_{1}+F_{2}\left(\frac{\partial \phi}{\partial n}\right)_{2}
\end{aligned}
$$

where $\xi$ is the dimensionless co-ordinate $\xi=\frac{x}{L / 2}$ (Figure 3 ), and:

$$
F_{1}=\frac{1}{2}(1+\xi) ; \quad F_{2}=\frac{1}{2}(1+\xi)
$$

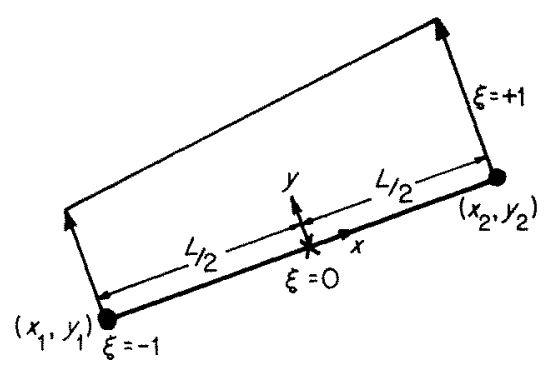

Figure 3. Conventional linear element

The singular point corresponding to a node $i$ is located at any arbitrary distance from $i$ and along the outward normal (Figure 4). Equation (12) for this singular point can now be

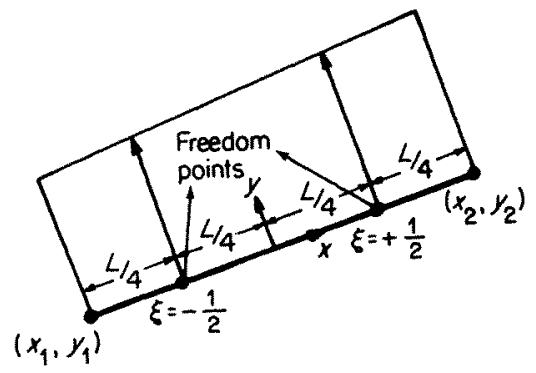

Figure 4. Non-conforming linear element 
written as:

$$
\sum_{j=1}^{N} \int_{S_{j}}\left(\begin{array}{ll}
F_{1} & F_{2}
\end{array}\right) \frac{\partial \phi^{*}}{\partial n} \mathrm{~d} S\left\{\begin{array}{l}
\phi_{1} \\
\phi_{2}
\end{array}\right\}=\sum_{j=1}^{N} \int_{S_{j}}\left(\begin{array}{ll}
F_{1} & F_{2}
\end{array}\right) \phi^{*} \mathrm{~d} S\left\{\begin{array}{l}
\frac{\partial \phi_{1}}{\partial n} \\
\frac{\partial \phi_{2}}{\partial n}
\end{array}\right\}
$$

or:

$$
\sum_{j=1}^{N}\left(h_{i, j-1}+h_{i, j}\right) \phi_{i}=\sum_{j=1}^{N}\left(g_{i, j-1}+g_{i, j}\right)\left(\frac{\partial \phi}{\partial n}\right)_{i}
$$

where:

$$
\left.\begin{array}{ll}
h_{i, j-1}=\int_{S_{j-1}} F_{1} \frac{\partial \phi^{*}}{\partial n} \mathrm{~d} S ; & h_{i, j}=\int_{S_{j}} F_{2} \frac{\partial \phi^{*}}{\partial n} \mathrm{~d} S \\
g_{i, j-1}=\int_{S_{i-1}} F_{1} \phi^{*} \mathrm{~d} S ; & g_{i, j}=\int_{S_{j}} F_{2} \phi^{*} \mathrm{~d} S
\end{array}\right\}
$$

That is, for each node an equation is set up taking into account the contributions made by the adjoining elements. This approach may give rise to problems mentioned in the 'Introduction'. To overcome these problems either the 'double-node' approach is followed along with a refined mesh near the points where there is a change in the nature of boundary conditions or as proposed in this paper, non-conforming (or discontinuous) linear elements may be employed whereby the freedoms of an element are not defined at the geometric nodes, i.e. at $\xi= \pm 1$ but at $\xi= \pm \frac{1}{2}$ (Figure 5). The interpolation functions take the form:

$$
F_{1}=\left(\frac{1}{2}-\xi\right) ; \quad F_{2}=\left(\frac{1}{2}+\xi\right)
$$

There are $2 N$ 'freedom points' defined over the whole boundary of the domain and the two equations for any point $i$, similar to equation (16) can be written as:

$$
\left.\begin{array}{rl}
\sum_{j=1}^{N}\left(h_{i, 2 j-1}\right) \phi_{i} & =\sum_{j=1}^{N}\left(g_{i, 2 j-1}\right)\left(\frac{\partial \phi}{\partial n}\right)_{i} \\
\sum_{j=1}^{N}\left(h_{i, 2 j}\right) \phi_{i} & =\sum_{j=1}^{N}\left(g_{i, 2 j}\right)\left(\frac{\partial \phi}{\partial n}\right)_{i}
\end{array}\right\}
$$

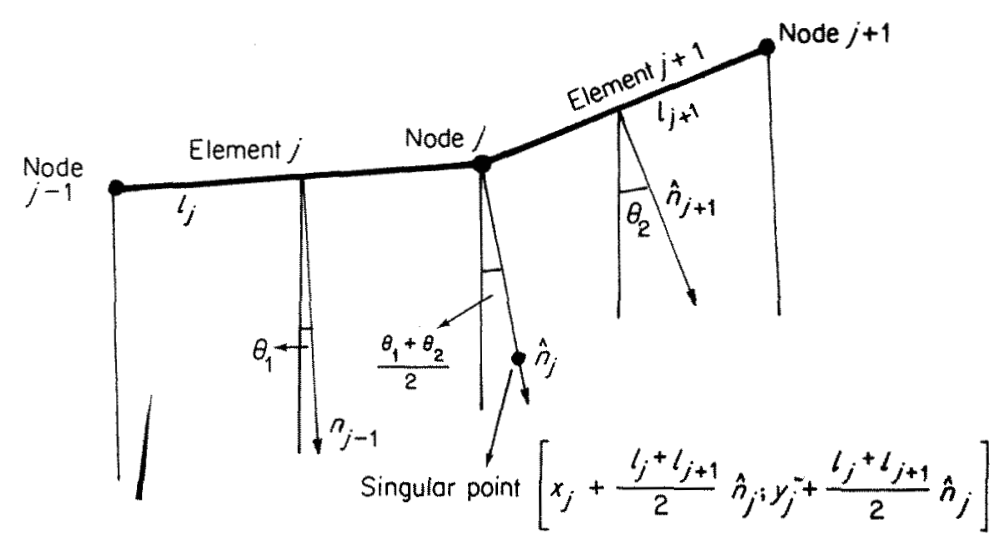

Figure 5. Location of singular point for linear element 
where:

$$
\left.\begin{array}{ll}
h_{i, 2 j-1}=\int F_{1} \frac{\partial \phi^{*}}{\partial n} \mathrm{~d} S ; & h_{i, 2 j}=\int F_{2} \frac{\partial \phi^{*}}{\partial n} \mathrm{~d} S \\
g_{i, 2 j-1}=\int F_{1} \phi^{*} \mathrm{~d} S ; & g_{i, 2 j}=\int F_{2} \phi^{*} \mathrm{~d} S
\end{array}\right\}
$$

Equation set (19) produces a $2 N \times 2 N$ system of equations which can be solved to give the solution on the boundary. Once the boundary solution is generated, solution in the interior can be obtained using equation (9) where $c^{i}=1$.

\section{APPLICATIONS}

Inviscid laminar flow around a circular obstacle in a channel

Consider the flow past an infinitely long cylinder positioned symmetrically between the two flat plates of infinite dimensions. We can choose the equivalent finite domain as shown in Figure 6. This is a typical fluid flow problem solved using the Finite Element Method with 55 nodes and 80 elements ${ }^{10}$ and 72 nodes and 110 elements. ${ }^{11}$ The problem has also been analysed using constant and linear boundary elements following the conventional Boundary Element Method. ${ }^{12}$

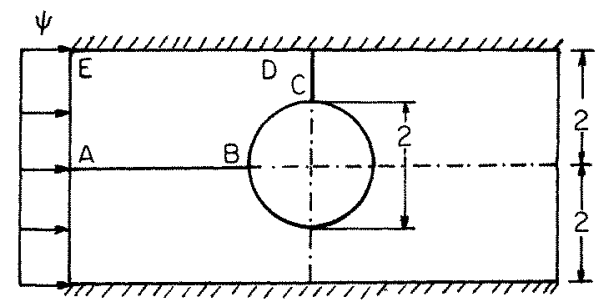

Figure 6. Flow past a circular obstacle in a channel

The governing equation for the problem is:

$$
\frac{\partial^{2} \psi}{\partial x^{2}}+\frac{\partial^{2} \psi}{\partial y^{2}}=0
$$

where $\psi$ is the stream function.

With properly specified boundary conditions it is possible to take only a quarter of the domain (Figure 7 ). In the stream function $(\psi)$ formulation, these boundary conditions are easily determined. The axis of symmetry and the upper boundary are both streamlines. The

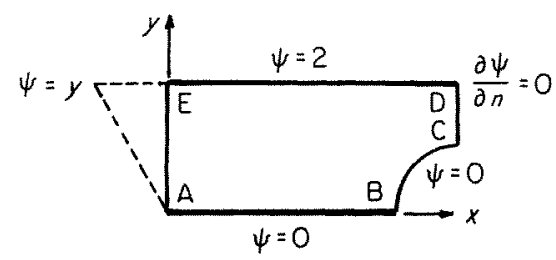

Figure 7. Boundary conditions for quarter domain 


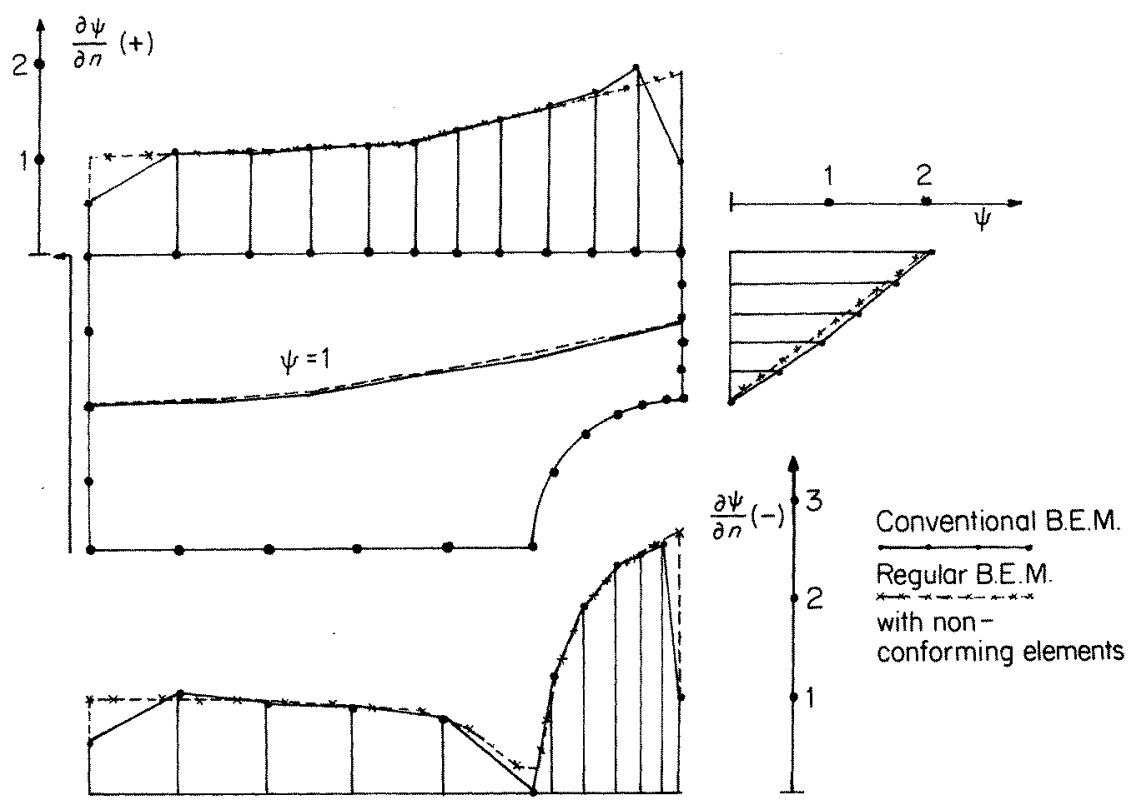

Figure 8. Computed values of $\psi$ and $\partial \psi / \partial n$ along the boundary using Conventional and Regular Boundary Element Methods

Conventional B.E.M.
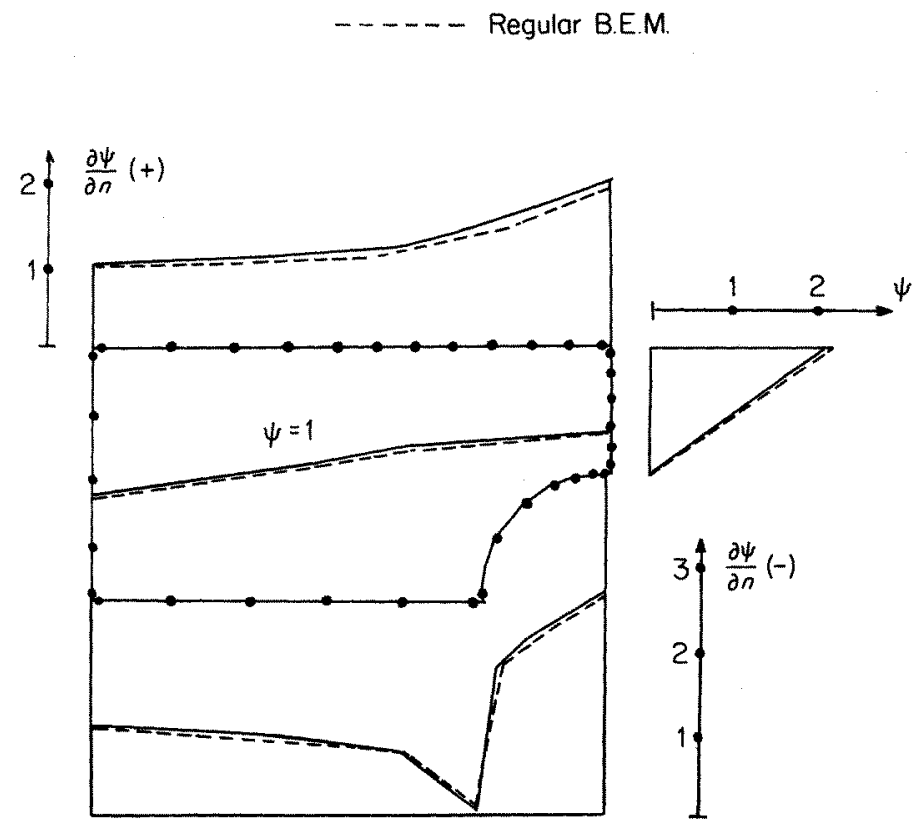

Figure 8(a). Computed values of $\psi$ and $\partial \psi / \partial n$ along the boundary using Conventional and Regular Boundary Element Methods, following the double-node approach 
axis of symmetry is a streamline because there is no flow across that line. The boundary of the cyclinder is also a streamline for the same reason. The arbitrary numerical values for streamlines fromed by the axis of symmetry and the cylinder, and the upper boundary are taken as $\psi=0$ and $\psi=2$ respectively.

The problem is solved using 32 linear boundary elements, and the boundary solutions thus obtained for 'Conventional' and 'Regular' Boundary Element Methods are compared in Figure 8. In the latter case non-conforming boundary elements have been employed. As is evident, the use of conforming elements tends to give good results for the $\psi$ and $\partial \psi / \partial n$ functions along the boundary but there is a distortion of the value of $\partial \psi / \partial n$ near those corners in which the normal derivative can take two different values depending upon the side under consideration. No such problem arises in the case of discontinuous elements. However, the problem can also be remedied by taking two nodes close to each other at the corners, one belonging to each side. ${ }^{12}$ This double-node approach has already been demonstrated using the 'Regular method' in Reference 3 by the authors and the results are reproduced in Figure $8(\mathrm{a})$ for the convenience of the reader. Streamline $\psi=1$, inside the domain is also plotted (Figure 8 ) to illustrate the solution in the interior which agrees well for the two methods.

It should be noted that for the 'Regular Method', these results correspond to the best position of the fundamental solution singularity, outside the domain and along the positive normal. This position was found to be one half the length of the element.

\section{Inviscid laminar flow in a channel past a disc}

Let $\psi$ be the stream function to represent the flow in a channel and let it be at normal incidence to the disc, as shown in Figure 9. Only a quarter of the domain needs to be

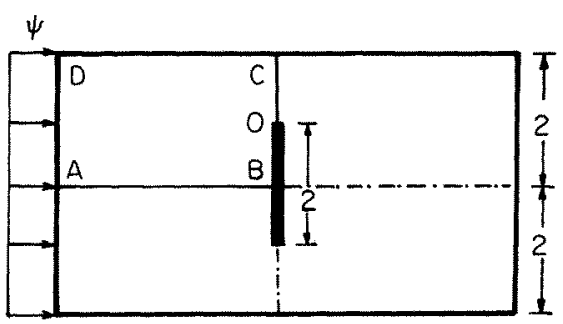

Figure 9. Flow past a disc in a channel

considered for analysis because of symmetry. The prescribed boundary conditions for the problem are shown in Figure 10. An infinite speed will be acquired by the stream at point ' $O$ ', the edge of the plate, giving rise to a singularity in the mathematical solution. ${ }^{13}$ The

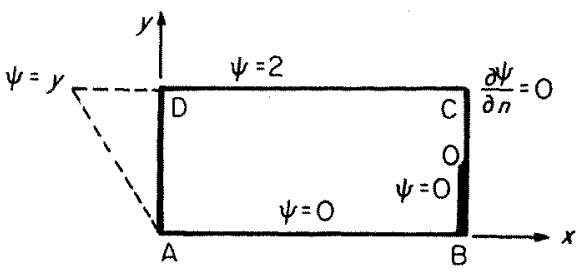

Figure 10. Boundary conditions for quarter domain 


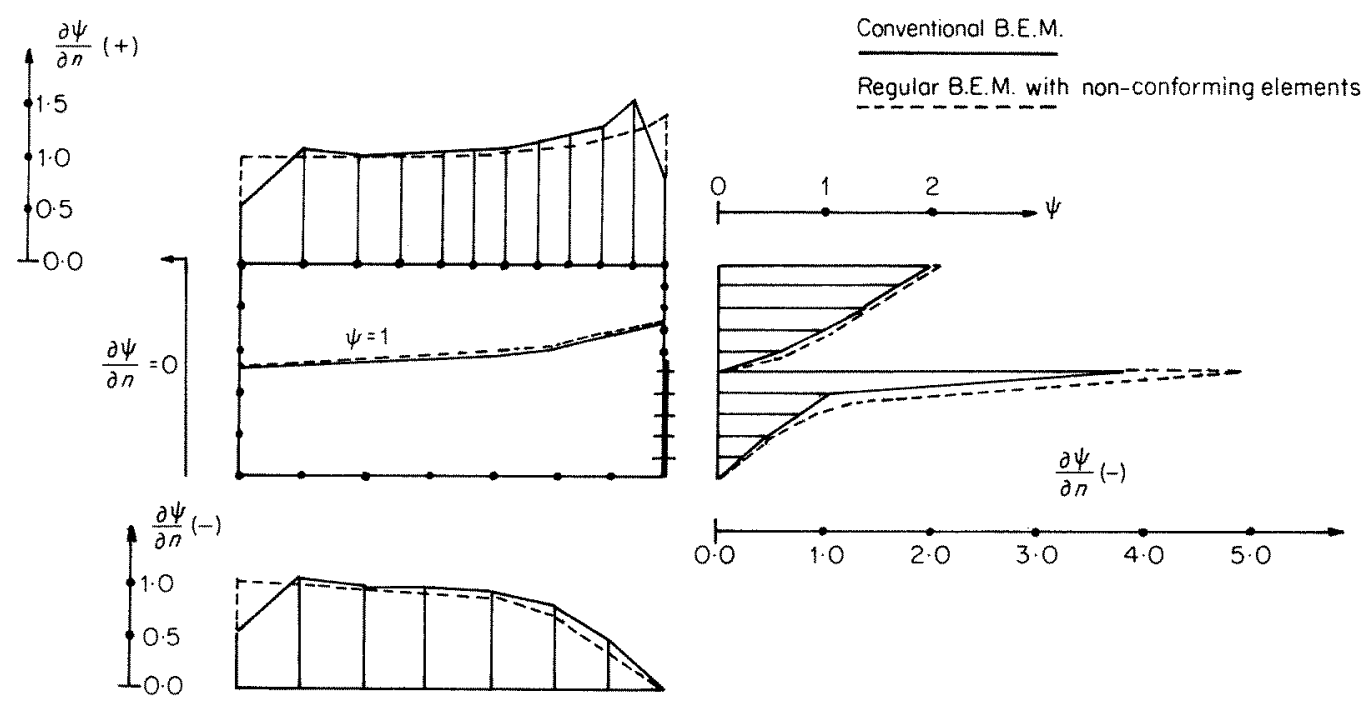

Figure 11. Computed values of $\psi$ and $\partial \psi / \partial n$ along the boundary using Conventional and Regular Boundary Element Methods

boundary of the quarter domain was divided into 32 linear elements with the same number of freedoms defined at the geometric nodes for continuous elements used in the Conventional Method and two freedoms over each discontinuous element employed in the case of the Regular Method.

The boundary solutions obtained for the two methods are compared in Figure 11, whereas Figure 11(a) shows the boundary solutions obtained by using the two methods, following the

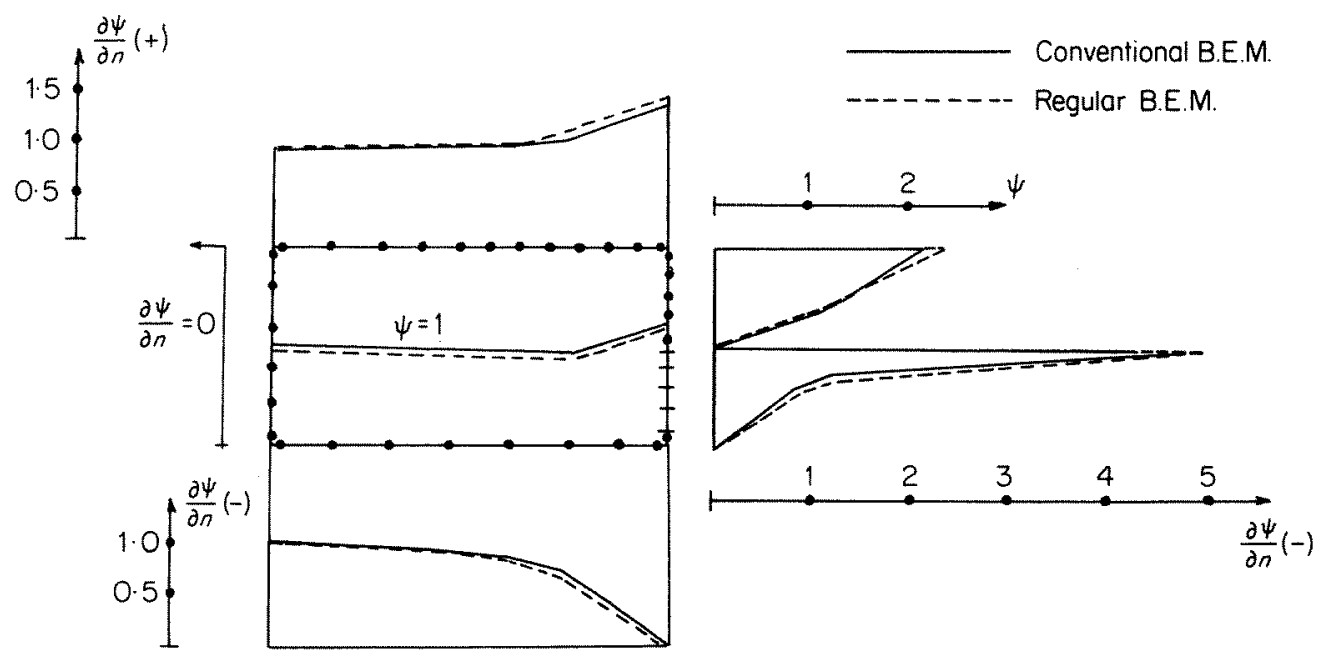

Figure 11(a). Computed values of $\psi$ and $\partial \psi / \partial n$ along the boundary using Conventional and Regular Boundary Element Methods, following the double-node approach 


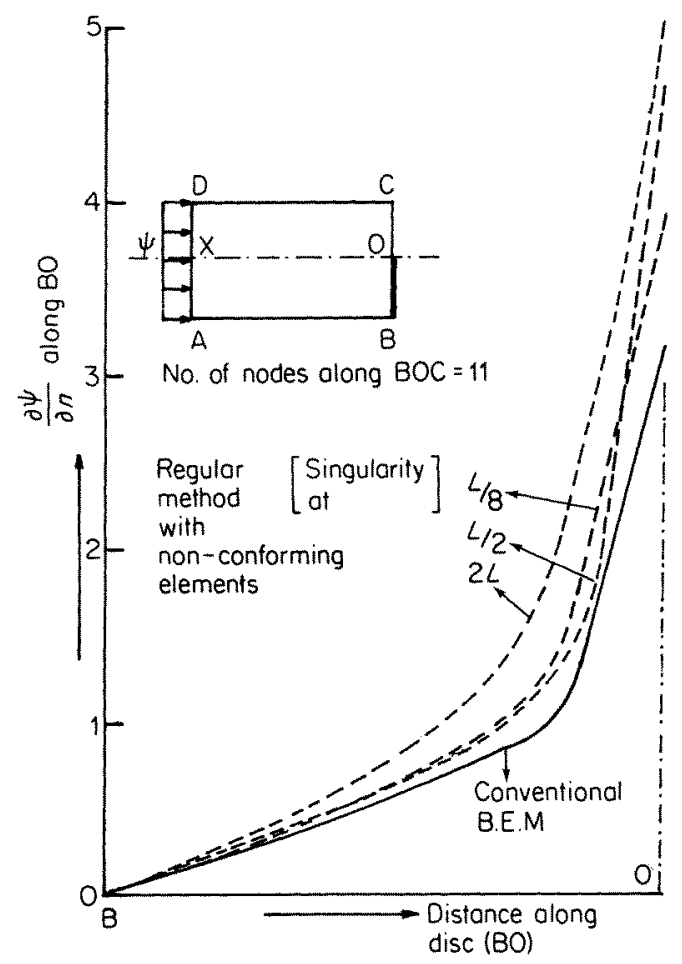

Figure 12. Variation of velocities $(\partial \psi / \partial n)$ along the boundary of the dise ( $\mathrm{BO})$ against location of fundamental solution singularity

'double-node' approach. Streamline $\psi=1$ is also plotted to illustrate the interior solutions. It should be noted that for the 'Regular Method' these results correspond to the best position of the fundamental solution singularity. A systematic study was undertaken to determine this position. It was observed that the quality of the solution deteriorated as the singularity was moved away from the domain of the problem. Best results were obtained with the singularity at one half the element length away from the element along the outward normal to the freedom node (Figure 12); but as shown in Figure 13, the interior solution is not affected much by changing this singularity location.

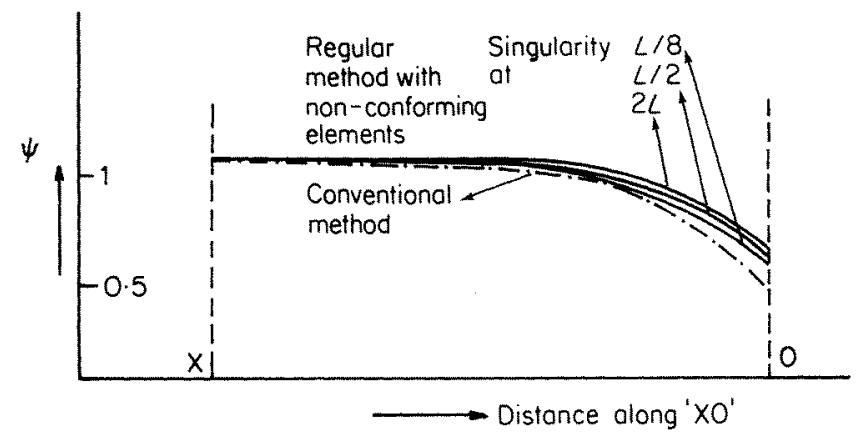

Figure 13. Internal solution against location of fundamental solutions singularity 


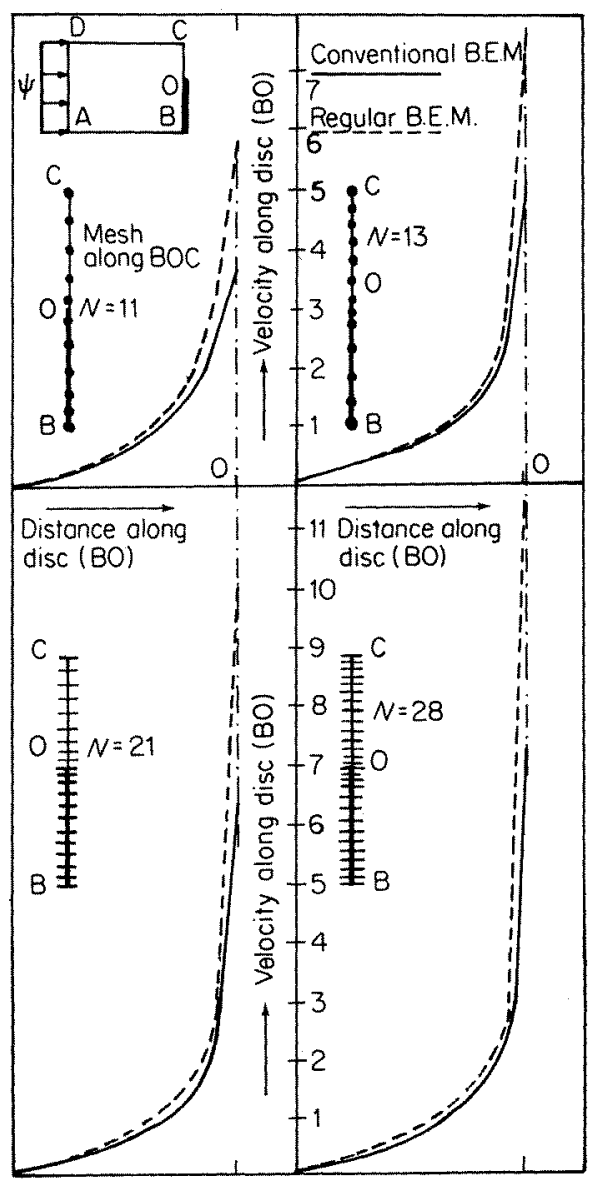

Figure 14. Quality of boundary solution generated by Conventional and Regular (with non-conforming elements) Methods for different boundary meshes employed in the neighbourhood of singular point

The quality of the solution generated by the two methods was also studied for different boundary meshes employed in the neighbourhood of the singular point (Figure 14). It has been shown that concentration of integration points around singularities is not a particular requirement when non-conforming elements are used but it may be necessary in the Conventional Method to avoid the quality of the solution being impaired even away from the singular points.

\section{CONCLUSIONS}

In this paper a 'Regular Boundary Element Method' has been presented and applied to harmonic fluid flow problems. The regular variant is obtained by locating the singular point of the fundamental solution outside the domain of the problem.

This method has the following advantages over the Conventional Boundary Element Method.

(a) The class of problems to which the Conventional Method may be applied is limited by the requirement that the singular boundary integrals do not diverge, the corresponding 
limitation of the Regular Method is substantially weaker because the kernels are everywhere regular over the boundary.

(b) Since no special attention is needed to handle singular kernels and the integrands are more slowly varying, the integrals in the regular method may be accurately determined at less computational cost than in the usual method.

It is also shown that non-conforming boundary elements may be used to overcome the modelling difficulties at singular boundary points (corners) and at points where abrupt changes in boundary conditions occur. The solution obtained using continuous elements is poorer in the neighbourhood of these anomalous points than their counterparts and this degradation may not be localized.

\section{REFERENCES}

1. L. C. Wrobel and C. A. Brebbia, 'The Boundary Element Method for steady state and transient heat conduction, in: Numerical Methods in Thermal Problems (Ed. R. W. Lewis and K. Morgan), Pineridge Press, 1980.

2. C. A. Brebbia and L. C. Wrobel, 'Steady and unsteady potential problems using the Boundary Element Method', in: Recent Advances in Numerical Methods in Fluids (Ed. C. Taylor and K. Morgan), Pineridge Press, 1980.

3. C. Patterson and M. A. Sheikh, 'Regular Boundary Integral Equations for fluid flow', in: Numerical Methods in Laminar and Turbulent Flow (Ed. C. Taylor and B. A. Schrefler), Pineridge Press, 1981.

4. C. Patterson and M. A. Sheikh, 'Regular Boundary Integral equations for stress analysis', in: Recent Advances in Boundary Element Methods (Ed. C. A. Brebbia), Springer Verlag, 1981.

5. J. C. Lachat, 'A further development of the Boundary Integral Technique for elastostatics', Ph.D. Thesis, University of Southampton, 1975.

6. C. Patterson and M. A. Sheikh, 'A Regular Boundary Element Method for coupled subdomains', in: Numerical Methods for Coupled Problems (Ed. R. W. Lewis), Pineridge Press, 1981.

7. C. Patterson, 'Sufficient conditions for convergence in the Finite Element Method for any solution of finite energy', in: Mathematics of Finite Elements and Applications (Ed. J. R. Whiteman), Academic Press, 1973.

8. C. Patterson and M. A. Sheikh, 'Discontinuous boundary elements for heat conduction', in: Numerical Methods in Thermal Problems (Ed. R. W. Lewis B. A. Schrefler), Pineridge Press, 1981.

9. C. Patterson and M. A. Sheikh, 'Non-conforming boundary elements for stress analysis', in: Recent Advances in Boundary Element Methods (Ed. C. A. Brebbia), Springer Verlag, 1981.

10. L. J. Segerlind, Applied Finite Element Analysis, Wiley, New York, 1976.

11. H. C. Martin, Finite element analysis of fluid flow', in: Matrix Methods in Structural Mechanics, AFFDL TR 68 150, Patterson Air Force Base (1969).

12. C. A. Brebbia, The Boundary Element Method for Engineers, Pentech Press, London, 1978.

13. L. M. Milne-Thompson, Theoretical Hydrodynamics, Macmillan, London, 1968. 